Originalveröggentlichung iln: Imhausen, Annette, Pommerening, Tanja (Hg.), Writings of Early Scholars in the Ancient Near East, Egypt, Rome, and Greece. Translating Ancient Scientific Texts (Beiträge zur Altertumskunde 286), Berlin - New York 2010, S. 201-218

\title{
Zur Neuedition des hieratisch-demotischen Papyrus Wien D 6257 aus römischer Zeit
}

\section{Friedhelm Hoffmann (Ludwig-Maximilians-Universität München)}

Der Wiener Papyrus, über den ich hier schreibe, ist kein Unbekannter. Er wurde bereits 1976 von Eva Reymond unter dem Titel A Medical Book from Crocodilopolis erstmals veröffentlicht. ${ }^{1}$ Meine Arbeit an der Neuedition dieses Textes gibt mir Gelegenheit, die Frage nach der Übersetzbarkeit antiker Wissenschaftssprachen in kritischer Auseinandersetzung mit der bisherigen Forschung am Wiener Papyrus zu behandeln.

Reymond stellt ihrer editio princeps zunächst eine Einleitung zur Wiener Papyrussammlung voran (S.21f.). Das ist verdienstvoll, da ein stimulierender Überblick über das höchst bedeutsame demotische ${ }^{2}$ Textmaterial in Wien sehr begrüßenswert ist. Reymond geht im weiteren Verlauf ihrer Einleitung u.a. noch speziell auf die Papyrusfunde im Fayum ein, der Gegend, in der die Hauptmasse der Wiener demotischen Papyri gefunden worden ist (S.23-30). Dabei skizziert sie auch mögliche Verbindungen zwischen griechischsprachiger hellenistischer Wissenschaft und den Wiener demotischen wissenschaftlichen Papyri aus dem 1. und 2. Jh. n. Chr.

Im nächsten Hauptteil ihres Buches (S. 31-40) wendet sich Reymond dem Wiener medizinischen Papyrus zu und beschreibt ihn, wie es üblich ist, hinsichtlich Material, Rekonstruktion, Erhaltung, Datierung, Paläographie und Sprache. Der Papyrus stammt aus der Zeit um 100 n. Chr. Im folgenden Abschnitt (S. 41-45) erhält der Leser eine „Analyse des Inhaltes“ der von Reymond rekonstruierten 16 Kolumnen. Sie hat den gesamten Text nach inhaltlichen Gesichtspunkten in

1 REYMOND (1976).

2 Die demotische Schrift ist eine um 650 v. Chr. entstandene ägyptische Kursive, die bis um $450 \mathrm{n}$. Chr. belegt ist. Allgemein zum Demotischen siehe DEPAUW (1997) und HOFFMANN (2000). Speziell zu den demotischen literarischen und paraliterarischen Texten in Wien vgl. REYMOND (1983). 
sechs Bücher eingeteilt, die eine je unterschiedliche Anzahl von Rezepten umfassen. Diese Einteilung stammt wohlgemerkt von Reymond, folgt aber natürlich der Reihenfolge der Rezepte, wie sie sich in ihrer Rekonstruktion auf dem Papyrus finden. Auf S. 45-63 beschreibt Reymond die einzelnen Abschnitte noch detaillierter. Mit einer Zusammenfassung und einem Ausblick auf die historische Bedeutung des Papyrus schließt der Einleitungsteil.

Dann folgt die Umschrift oder genauer: eine erste Umschrift des Papyrus, die Reymond in die groben Umrisse der Fragmente einfügt (S. 65-80). Der Text ist sehr fragmentiert, was die Bearbeitung natürlich erschwert - vor allem da, wo die Löcher größer als das Erhaltene sind. Ab S. 81 folgt erneut eine Umschrift, bei der die Umschrift und Übersetzung stets auf einer Doppelseite einander gegenüberstehen. Überschriften mit der Angabe der Kolumne und des Inhaltes gliedern den Text. Auf diesen Teil folgt der paläographische und philologische Kommentar, der zeilenweise angelegt ist (S. 133-197).

Den Abschluss der Edition bilden verschiedene Indizes, zunächst ein „Demotic Glossary“ (S. 201-242). Die Wörter werden durchgezählt, in Normalschreibung abgebildet, übersetzt und die Belegstellen angegeben. Im Falle von medizinischen Fachwörtern unterbleibt das hier. Dann wird lediglich auf den entsprechenden Eintrag im Index medizinischer Termini verwiesen. So ist es auch bei Drogennamen: Mit „Ph.“ für „Pharmakon“ und der entsprechenden Nummer wird lediglich auf das Drogenregister (S. 243-288) verwiesen, das ausführlicher gestaltet ist, indem zusätzlich die älteren ägyptischen Entsprechungen der Wörter und die jüngeren, d.h. koptischen Formen der Drogennamen aufgeführt werden, dazu noch griechische und lateinische Entsprechungen derselben Pflanzen oder Mineralien. Reymonds Band wird durch Fototafeln ergänzt, auf denen sie den Papyrus vollständig abbildet.

Bis hierher habe ich die Perspektive des Lesers oder Benutzers von Reymonds Edition eingenommen. Man muss nun feststellen, dass der Wiener Papyrus trotz seiner großen Bedeutung gerade auch für die Frage nach wissenschaftlichen Kontakten zwischen ägyptischer, griechischer sowie - von Reymond nicht gesehen - mesopotamischer Medizin kaum rezipiert worden ist. Und das liegt leider an der Qualität der Edition. Wolfhart Westendorf drückt das in seinem Handbuch der altägyptischen Mediz̨in zurückhaltend so aus: „Wegen der vielen Lücken und der mitunter unzulänglichen Herausgabe zunächst nur bedingt für 
die Medizingeschichte benutzbar". 3 Wechseln wir also die Perspektive und schreiten gemeinsam einmal den Weg vom Papyrus zur Edition ab. Das gibt Gelegenheit, in Auseinandersetzung mit Reymond meine Forderungen an die Edition eines spätägyptischen medizinischen Textes wie des vorliegenden anschaulicher $\mathrm{zu}$ formulieren.

An erster Stelle steht das Aufspüren zugehöriger Fragmente. Eine Nachsuche in Wien hat 2008 nur drei kleine Fragmente zutage gefördert, die Reymond übersehen hatte. Aber genauso wichtig für die Textrekonstruktion ist auch das Ausscheiden nicht zugehöriger Stücke. Davon sind Reymonds Kolumnen $x+I$ und $x+I I$ betroffen. Sie tragen Anweisungen zur Textilfärberei, haben mit ägyptischer Medizin also nichts zu tun. ${ }^{4}$ Damit fallen die ersten beiden Kolumnen in Reymonds Textrekonstruktion weg. Aber auch die vermeintliche dritte ist zu streichen, denn sie ist völlig leer. Es ist mir ein Rätsel, wie Reymond darin etwas anderes als den freigelassenen Schutzstreifen vor der ersten Kolumne der Rolle sehen konnte. Reymonds Kolumne $\mathrm{x}+\mathrm{IV}$ ist also die wirkliche erste Kolumne des medizinischen Textes, für den somit nicht 16, sondern nur 13 Kolumnen übrigbleiben.

Aber auch diese Zahl schrumpft weiter, wenn man erkennt, dass sich viele Fragmente, die von Reymond als eigenständige Kolumnen gewertet werden, noch zusammenfügen lassen. Mitunter gehören vier von Reymond getrennt gesehene Stücke in Wirklichkeit zusammen. Am Ende erhalten wir sechs Kolumnen, Reymonds Kolumnenzählung ist damit überhaupt nicht mehr zu retten. Ich habe den Text daher neu durchgezählt und in einer umfangreichen Konkordanz alte und neue sowie neue und alte Zählung miteinander korreliert. ${ }^{5}$

Doch kommen wir nun zum Text. Reymond hat zwei Dinge in Umschrift und Übersetzung nicht wiedergegeben. Erstens sind im Original nämlich die Paragraphenanfänge konsequent rot geschrieben.

3 WeStENDORF (1999): 55, Anm. 72.

4 Erkannt von QUACK (1999): 456. Die Handschrift ist zwar ähnlich, aber Reymond hätte stutzig werden müssen, weil die Überschriften in diesem Bereich mit $p 3$ g3y ... „Die Art ...“, also „Die Anweisung, dies und das zu tun", ganz anders sind als im medizinischen Papyrus, wo es nie so, sondern fast immer mit „Heilmittel“ oder „Ein anderes“ losgeht. Joachim Friedrich Quack bereitet eine Neubearbeitung des Färbetextes vor.

5 Weniger dramatisch wirkt sich aus, dass Reymond bzw. der Papyrusrestaurator den Papyrus noch nicht vollständig geglättet und zurecht gezogen hatte, als Reymond ihn publizierte. 
Diese Information geht bei Reymond verloren. ${ }^{6}$ Ich meine, die Rubrizierung als vom antiken Schreiber angewandtes optisches Gliederungsmittel muss in Umschrift und Übersetzung adäquat berücksichtigt werden. In der Ägyptologie nimmt man dazu gerne die Unterstreichung. Das geht bei der Übersetzung vielleicht noch an, auch wenn es eine satztechnische Sünde ist. Aber bei der Umschrift halte ich dieses Verfahren für weniger geeignet, da es ohnehin schon genug Zeichen mit Punkt, Bogen oder Strich darunter gibt, so dass die Lesbarkeit beeinträchtigt wird. Ich nehme daher lieber eine hellgraue Unterlegung. ${ }^{7}$ Für wichtig halte ich es, die Rubren in Umschrift und Übersetzung kenntlich zu machen, weil ich finde, auch die Übersetzung sollte möglichst alle Informationen über den bearbeiteten Text bieten, da gerade Nichtägyptologen hauptsächlich mit der Übersetzung arbeiten werden. Außerdem müssen Unsicherheiten der Umschrift und der Übersetzung klar als solche gekennzeichnet werden.

Auch Spatien im Text sollten angegeben werden. Denn oft haben sie eine vom Schreiber beabsichtigte gliedernde Funktion. ${ }^{8}$ Das geht im Wiener Text zwar, soweit ich sehe, nicht glatt auf. Aber wenn man die Spatien wie Reymond von vornherein gar nicht in Umschrift und Übersetzung wiedergibt, verbaut man sich den Weg, ihren Zweck zu erforschen. Ich gebe Spatien durch || wieder.

Und noch etwas fällt in Reymonds Edition unter den Tisch. Der Wiener Papyrus ist nämlich nicht rein demotisch geschrieben, sondern mischt hieratische und demotische Schrift. ${ }^{9}$ Was nun die Verwendung der beiden Schriften im Wiener Papyrus anbelangt, so erfolgt der Wechsel zwischen den beiden Schriften sehr kleinräumig. Es ist keineswegs so, dass komplett hieratisch geschriebene mit demotisch geschriebenen Rezepten abwechseln. Vielmehr findet sich in einem einzigen

6 Da sie offenbar mehr von ihrer eigenen Umschrift als vom Original übersetzt hat, führt dies dazu, dass Reymond zuweilen auch mit schwarz geschriebenen Wörtern eine Rezeptüberschrift beginnen lässt (z. B. mit $n 3$ hnyn.w [d.i. n3 hnyn.w] in $\mathrm{x}+\mathrm{VIII} .15$ [d.i. 2.15]). Das bringt natürlich die ganze Übersetzung durcheinander.

7 Im vorliegenden Aufsatz ersatzweise Fettdruck.

8 Vgl. z. B. LIPPERT (2004).

9 Hieratisch nennt man die seit dem 3.Jt.v. Chr. existierende ägyptische Schreibschrift, die auch nach der Entstehung der demotischen Schrift in der Mitte des 7. vorchristlichen Jahrhunderts nicht ausstirbt. 
Rezept Hieratisch neben Demotisch, ja teilweise wird sogar innerhalb eines Wortes gewechselt. Natürlich bedeuten z. B. hieratisch geschriebenes sriw und demotisches sly dasselbe, nämlich „Husten“, und es ergibt sich kein inhaltlicher Unterschied, ob man etwa das Verb "geben" hieratisch oder demotisch schreibt, zumal die unterschiedlichen Schreibungen teilweise sogar in denselben Phrasen vorkommen. Aber notiert man den Unterschied in Umschrift und Übersetzung nicht, nivelliert man den Text in einer Weise, die ihn weitgehend seiner textgeschichtlichen Dimension beraubt oder diese zumindest verschleiert. Denn wenn auch ganz banale Wörter wie „geben“ oder „bei“ im Wiener Papyrus in beiden ägyptischen Schriften nebeneinander vorkommen, dann bedeutet das doch mit der allergrößten Wahrscheinlichkeit, dass sich hier Eigenheiten der Vorlage oder Vorlagen erhalten haben, von der oder denen der Wiener Papyrus eine unmittelbare oder mittelbare Abschrift ist. Es ist nämlich nicht plausibel, dass ein Schreiber einfach so einmal die eine und einmal die andere Schrift verwendet. Selbst wenn man mir hier nicht folgen wollte, müsste man auf der drucktechnischen Unterscheidung von hieratischer und demotischer Schrift in einer modernen Ausgabe bestehen, gerade um die Frage nach Ursache oder Zweck dieser Mischung angehen zu können.

Ägyptologen geben Hieratisches in der Umschrift gerne durch Hieroglyphen wieder, Demotisches aber in lateinischen Buchstaben. ${ }^{10}$ Als Problem dabei sehe ich, dass die hieratischen Wörter so eigentlich gar nicht umschrieben werden. Das verbaut Nichtägyptologen die Verbindung zum natürlich alphabetisch sortierten Wortregister. Denn man muss jetzt immer noch wenigstens Hieroglyphen lesen können. Ich habe mich daher entschlossen, auch hieratische Wörter oder Wortteile in lateinischer Umschrift wiederzugeben, aber im Unterschied zu demotischen Wörtern in Kapitälchen. Die Umsetzung in Hieroglyphen wird der Leser im Wortregister ebenfalls finden.

Bei der Gestaltung von Umschrift und Übersetzung ist meiner Meinung nach noch ein weiterer Punkt zu berücksichtigen, nämlich die inhaltliche Erschließung. Reymond fügt dazu Zwischenüberschriften ein, aus denen hervorgeht, wo ein Rezept beginnt und wogegen es ihrer Meinung nach wirken soll. Ein derartiger Hinweis für den Leser ist sicher sinnvoll, vor allem, wenn es sich um nur trümmerhaft

Z. B. VOS (1993). 
erhaltene Abschnitte handelt. Die Herausgeber des Grundrisses der Medizin $\operatorname{der}$ Alten Agypter ${ }^{11}$, in dem ausschließlich die älteren ägyptischen medizinischen Texte bis etwa $1000 \mathrm{v}$. Chr. erfasst sind, haben ja sogar die Originaltexte in Einzelabschnitte zerlegt und diese nach inhaltlichen Kriterien neu geordnet. So sind z.B. alle Hustenrezepte aus verschiedenen Papyri zusammengetragen, dann Rezepte gegen diese, dann gegen jene Krankheit. So hat man zwar bequem sämtliche aus heutiger medizinischer Sicht zusammengehörigen Texte beieinander, aber die Organisation der Originalquellen wird dabei unterdrückt. Ich halte diese aber für wichtig. Denn nur so wird nicht nur sichtbar, dass die Ägypter dies oder jenes gekannt und behandelt haben, sondern auch, in welchem Zusammenhang ihre Rezepte stehen. Selbst wenn sich herausstellen sollte, dass dieser Zusammenhang in einer Sammelhandschrift wie dem Wiener Papyrus rein zufällig ist, wäre das ein Erkenntnisgewinn. Außerdem ist die Edition eines einzigen Papyrus etwas anderes als die eines Kompendiums zur ägyptischen Medizin.

Gleichwohl hat die Durchnummerierung der einzelnen Textabschnitte, wie sie im GRUNDRISS vorgenommen wird, ihre rein praktischen Vorteile. Ich bin daher dafür, den Wiener Papyrus mit zwei voneinander unabhängigen Zählungen zu versehen. Eine sollte eine Paragraphenzählung sein. Wenn man schon Überschriften einfügt, dann ist es meiner Meinung nach am einfachsten, dort auch gleich eine Paragraphenzahl hinzusetzen. Sie erleichtert dem Leser die inhaltliche Erschließung des Textes.

Die zweite, davon unabhängige Zählweise ist die übliche Kolumnen-Zeilen-Zählung, die es erlaubt, z.B. auf der Fototafel gezielt die Schreibung eines Wortes oder ein grammatikalisches Phänomen zu überprüfen. Damit die Kolumnen- und Zeilenzahlen nun aber nicht im fortlaufenden Text untergehen, ${ }^{12}$ setze ich diese Zählung als Marginalie. ${ }^{13}$ Die genaue Stelle des Zeilenwechsels ist in der Umschrift durch | bezeichnet, genauso in der Übersetzung. Ich betone dies, weil es z. B. in der griechischen Papyrologie weithin Usus ist, die Übersetzungen ganz ohne Zeilenzahlen zu lassen, vermutlich um anzuzeigen, dass die Übersetzung ohnehin nur als Krücke für diejenigen gedacht ist, die

11 Speziell GRUNDRISS IV, 1 und V.

12 Vgl. Hoffmann (1996): 679-680, Anm. 2.

13 Im vorliegenden Aufsatz nicht realisiert. 
kein Griechisch können. Aber für wen mache ich denn die Übersetzung eines ägyptischen medizinischen Textes? Doch nicht zuletzt für Medizinhistoriker, damit sie mit Problemen weiterkommen, die ich als Ägyptologe nicht lösen kann.

Ärzte werden wohl weniger als Leser in Frage kommen. Ich würde ihnen auch nicht so weit entgegenkommen, dass ich z. B. Otitis media purulenta statt „Eiterfluss aus dem Ohr“ übersetzen würde. Außerdem ist der moderne Fachterminus an die moderne Medizin gekoppelt. Er gehört zu ihrem Begriffssystem. Seine Verwendung in der Übersetzung eines antiken Textes sehe ich nicht nur als Anachronismus an, sondern geradezu als Vortäuschung einer im Original nicht gegebenen Kategorisierung. Ägyptologisches Übersetzen und medizinisches Deuten sind zweierlei. Wir müssen der Tatsache Rechnung tragen, dass wir vieles nicht sicher verstehen, dass aber auch die Grade der Sicherheit bzw. Unsicherheit unterschiedlich sind. Eine präzise moderne Terminologie kann das nicht abbilden, da sie quasi per definitionem nicht in der Lage dazu ist, Unwissenheit zu transportieren. ${ }^{14}$ Genau das sollen aber Umschrift und Übersetzung leisten. Sie sollten daher möglichst alle Informationen des Originals und möglichst alles, was wir wissen und nicht wissen, wiedergeben.

Als Ideal schwebt mir vor, jedes ägyptische Wort konsistent durch immer ein und dasselbe deutsche Wort wiederzugeben, sofern die Verständlichkeit darunter nicht leidet. Die berühmte Bibelübersetzung von Buber und Rosenzweig ${ }^{15}$ liegt auf derselben Linie. Und was bei einem literarischen Text funktioniert, geht bei einem wissenschaftlichen Text mit seiner viel stärker formalisierten Sprache erst recht. Unser Ziel muss es doch sein, zur ägyptischen Begrifflichkeit vorzustoßen und sie nicht im Interesse einer glatten Lesbarkeit der deutschen Begrifflichkeit

14 Am Rande sei erwähnt, dass, soweit ich sehe, auch die moderne Übersetzungswissenschaft - vgl. z. B. GERZYMISCH-ARBOGAST und MUDERSBACH (1998) und BAUMANN (2009) - in ihren Modellen nicht berücksichtigt, dass man als Altertumswissenschaftler beim Übersetzen ständig mit Wissenslücken umgehen muss. Ägyptologische Übersetzungen sind daher weniger Ergebnis eines operationalisierbaren, zielgruppenorientierten Übersetzungsprozesses als vielmehr ein notwendiger Teil der Forschungsbemühungen um das Verständnis einer alten Kultur.

15 Mir war zugänglich BUBER/ROSENZWEIG (1979-1985). Beachte auch die Beilage zum ersten Band: BUBER, Martin. 1979. Zu einer newen Verdeutschung der Schrift. 
zu opfern. Wir wollen doch die ägyptischen Konzepte verstehen - das ist den Preis einer unter Umständen unschönen deutschen Wiedergabe wert, finde ich.

Dabei ist freilich eine Einschränkung zu machen: Wenn die Möglichkeit besteht, dass ein Leser ohne ägyptologische Kenntnisse die Übersetzung falsch verstehen würde, sind helfende oder klärende Anmerkungen erforderlich. Das macht die Lektüre vielleicht schwieriger, aber sozusagen genauer und beugt jedenfalls Informationsverlust vor.

Wie wenig normierende Eingriffe angebracht sind, zeigt auch der Gebrauch verschiedener Tempora im Wiener Papyrus. Dazu ein Beispielrezept (2.x+13-3.4):

Ein an[Deres] Heilmittel des Puderns: [jeglichen(?) Abszess(?)] zugrundegehen lassen: (Hier kommen zunächst zwei Drogen mit Gewichtsangaben; anschließend heißt es weiter:)

FEIN ZERREIBEN; auf sie geben, nachdem du [ihn ein ARZTmittel hast machen zu las $]$ sen $[\ldots \ldots .$.$] vorher. Du sollst (3.2) die Abszesse salben (mit)$

Menschenmilch, [a]uf(s) Fe[uer] gestel[lt]. ||

Du sollst ihn mit dem trockenen HEILMITTEL pudern [... ... . ... Du sollst (3.3) d[ie] Abszesse < mit > ei[ner Na]del (auf)stechen, <um> zu erkennen, ob Blut in ihnen ist. (Der Text fährt dann noch mit weiteren Anweisungen fort.)

Im vorliegenden Rezept wird von den Infinitiven ${ }^{16}$ „fein zerreiben“ und ,geben" zum 3. Futur in der 2. Person Singular maskulinum gewechselt: „Du sollst dies und das tun“. Diese Verbform ist typisch in demotischen Anweisungen z.B. auch in mathematischen Prozedurtexten. In anderen Abschnitten wechseln Infinitive mit Konjunktiven der 3. Person Plural, die für unpersönliches „man“ steht (4.36-4.38):

HeIlmittel für einen Mann, der krank ist, || indem er ,ko[cht](?) $[\ldots . . .$.

Mönchspfeffer, $1 / 4 ; \mid$ |

n3y-Körniges, $1 / 4 ; \mid$ |

btt-Pflanze, 1/8; ||

Klee;

1 Gall $<\mathrm{e}>$.

16 Angesichts einiger demotischer Schreibungen wie di.t ,geben“, die auf den Infinitiv festgelegt sind und die in derselben Verwendungsweise in der Handlungsanweisung vorkommen, erscheint es mir am sinnvollsten, alle unmarkierten Formen als Infinitive zu verstehen, da die Ägypter selbst sie in der Spätzeit offenbar so aufgefasst haben und nicht mehr als subjektlose Passivformen nach dem Schema ,werde zerrieben“ etc. 
Und man soll sie (= die Galle) machen mit (4.37) Durchbohrung, zweimal. ||

Eselsurin, 4 [1/2(?)] Lok.

(Ein)kochen [bis] au[f] 4 [Lok]; (vom Feuer) nehmen. Und man soll sie (Pl.) wieder zerstampfen. || Und man soll (dazu)ge[ben]

HONIG, 1/30;

Öl, 1/8;

(4.38) Rindermilch, 2 Lok. ||

IN seinen Af[ter] GIESSEN. [

Man könnte noch mehr Beispiele für diese und ähnliche Unterschiede anführen. Wichtig ist die Erkenntnis, dass Struktur und Formulierung eine Reihe von Varianten aufweisen. Darin zeigt sich, dass der Wiener medizinische Papyrus eine Rezeptsammlung ist, in die Rezepte aus verschiedenen Quellen zusammengeflossen sind, wobei deren strukturelle und sprachliche Eigenheiten nicht oder höchstens in beschränktem Maße angetastet und vereinheitlicht worden sind.

Diese ganze sprachliche Vielfalt der Formen muss meiner Meinung nach auch in der Übersetzung stehenbleiben. Wir dürfen die Übersetzung nicht auf den Inhalt beschränken, sondern müssen, so gut es geht, auch die Form berücksichtigen. Das gilt, denke ich, für alle sprachlichen Phänomene, erst recht für solche, die auch noch sprachgeschichtliche Relevanz besitzen. Hierzu gehört - um ein auch den Nichtägyptologen leicht nachvollziehbares Beispiel zu wählen - der Gebrauch des Artikels, der eine sprachgeschichtlich jüngere Erscheinung ist. Eine Überschrift wie diese (2.15-2.16):

tys-Gefäß/-Flüssigkeit: aufhören lassen frische Falten(?) und die Störungen, die [... ... von] einem Kind (2.16) und [die] Wunden, die Störungen, welche geschehen $<a n>$ den Ellbogen und(?) [........... Wunde auch

mit inkonsequenter Artikelsetzung erweist sich damit als sekundär erweitert. Ein alter Überschriftenkern „tys-Gefäß/-Flüssigkeit: aufhören lassen frische Falten(?)" - ohne Artikel - ist später durch eine Aufzählung weiterer Krankheiten spezifiziert bzw. erweitert worden in einer jüngeren Sprachform mit Artikel. Um den Sprachstand in dieser Hinsicht möglichst exakt wiederzugeben, setze ich, wenn im Original kein Artikel steht, er aber (anders als im zitierten Beispiel) im Deutschen unverzichtbar ist, in der Übersetzung runde Klammern um den Artikel und kennzeichne ihn so als erläuternden Zusatz von mir. 
Doch eine Übersetzung sollte nicht nur korrekt sein. ${ }^{17}$ Dabei stellen eine besondere Schwierigkeit die ägyptischen Fachtermini dar, also vor allem die Krankheitsbezeichnungen und die Drogennamen, weniger die Körperteilbezeichnungen, da diese oft auch in Texten anderer Gattungen vorkommen und daher generell besser bekannt sind. Speziell bei den Krankheitsbezeichnungen hat man das Problem, dass mitunter quasi ,normale ägyptische Wörter eben auch als Fachtermini vorkommen. Natürlich kann man ein Wort wie $\underline{d n b}$ als „Verkrümmung“ oder $n s ̌ n$ als „Wüten“ übersetzen. Aber damit ist noch wenig darüber gesagt, welche Krankheit - nach unserem Verständnis - sich dahinter verbirgt, wenn es sonst keine Anhaltspunkte dafür im Text gibt. In solchen Fällen setze ich dann die wörtliche Übersetzung in Anführungszeichen, um auf den nicht normalen, sondern fachsprachlichen Gebrauch des Wortes hinzuweisen.

Besonders ausgeprägt sind die Übersetzungsunsicherheiten bei den Drogennamen. Es gibt hinsichtlich ihrer mineralogischen oder botanischen Identifizierung ganz unterschiedliche Grade an Sicherheit oder Unsicherheit. Manche Wörter sind auch aus anderen Texten bestens bekannt wie z.B. $m w$ „Wasser" ${ }^{\star 18}$ oder bi.t, demotisch iby „Honig“"19. Sie erscheinen in meiner Übersetzung daher ohne weitere Markierung. Bei anderen Wörtern gibt es zwar eine gängige ägyptologische Übersetzung, sie sagt aber unter Umständen wenig über die naturwissenschaftliche Seite aus z.B. sntrr „Weihrauch“20. Hier ist wenigstens im Drogenregister eine Klarstellung erforderlich, was dahintersteckt, nämlich das Harz von Boswellia-Arten ${ }^{21}$.

Bei wieder anderen Wörtern gibt es zwar eine übliche Übersetzung, aber auch kritische Stimmen dazu. Ein Beispiel dafür ist wr3 „Wicke“22. Erstens ist überhaupt nicht klar, auf welche der vielen Wickenarten man sich eigentlich festlegen soll. Manche Forscher haben überdies die Meinung vertreten, dass es sich um die Langbohne (Vigna unguiculata)

17 Übrigens weist Reymonds Bearbeitung gerade auch hier viele Defizite auf. Das muss ich hier nicht detailliert vorführen; vgl. dazu beispielsweise DEVAuCHELLE/PEZIN (1978).

18 Wв II: 50-53; GRUNDRISS VI: 225-233; ERICHSEN (1954): 154-155.

19 WB I: 434; GRUNDRISS VI: 156-168; ERICHSEN (1954): 26.

20 Wв IV: 180-181; GRUNDRISS VI: 449-454; ERICHSEN (1954): 440-441.

21 GERMER (1985): 110, 112.

22 ERICHSEN (1954): 93. 
handelt. ${ }^{23}$ Hier ist also ebenfalls eine Diskussion des Für und Wider angebracht. Und in der Übersetzung sollte „Wicke“ auf jeden Fall mit Fragezeichen stehen.

Aber bei allen botanischen und mineralogischen, seltener auch zoologischen Bestimmungen sollte man sich darüber im Klaren sein, dass die ägyptischen Bezeichnungen ${ }^{24}$ nicht mit den modernen naturwissenschaftlichen Artgrenzen oder Mineralklassifikationen übereinstimmen müssen bzw. es in der Regel auch wirklich nicht tun. ${ }^{25}$ Dennoch sollte man meiner Meinung nach in der Diskussion zur möglichst genauen Identifizierung stets den wissenschaftlichen Namen dazusetzen, um unmissverständlich klar zu machen, welches Tier, welche Pflanze oder welche Substanz man als Übersetzer meint. Die internationalen Namen haben außerdem den Vorteil, unabhängig von den modernen Einzelsprachen verständlich zu sein. „Unechtes Tännelkraut“ z.B. findet ein englischer Muttersprachler nicht in einem handelsüblichen deutsch-englischen Wörterbuch, kann es natürlich auch in einem ihm leicht zugänglichen englischen Pflanzenbuch nicht finden.

Im Wiener Papyrus kommen auch fremdsprachige Drogennamen vor, griechische und semitische sicher, vielleicht noch andere. Hier potenzieren sich die Probleme. Schon die Gleichsetzung eines griechischen Wortes mit einer demotischen Schreibung kann erhebliche Probleme bereiten. pprs = péperis „Pfeffer“ ist sicher. Aber ist qstryn oder qstrny identisch mit kassitérinos „aus Zinn Gemachter“ = ,Zinnpulver" o. ä., wie Reymond dachte, oder nicht doch eher mit kastór(e)ion „Bibergeil"? Aber selbst bei den auf der Wortebene klaren Entsprechungen kann es sich um griechische Wörter handeln, deren naturwissenschaftliche Bestimmung unsicher ist. Dann sollte man so ehrlich sein, das in der Übersetzung auch auszudrücken, gleichzeitig aber das, was gesichert erscheint, klarmachen. So habe ich mich im Falle von qrkhnts und qrkhts dazu entschieden, die griechischen Entsprechungen und deren im groben bekannte chemische Identifizierung anzugeben, indem ich „chálkanthos-Vitriol“" und „chalkîtis-Vitriol“ schreibe.

23 Devauchelle/Pezin (1978): 61 und Germer (1985): 88.

24 Es sei noch auf das Problem hingewiesen, dass die ägyptischen Wörter nicht über die Jahrhunderte hinweg konstant geblieben sein müssen. Einerseits kommen neue Bezeichnungen für dieselben Pflanzen auf, andererseits können im Laufe der Zeit dieselben Namen auf andere Pflanzen übertragen werden.

25 BAUM (1999). 
Im Zusammenhang mit den Drogen gibt es, wenn ansonsten alle Stricke reißen, nur einen einzigen Anhaltspunkt, die sogenannten Determinative. Mit diesen Zeichen wird am Ende eines Wortes angegeben, zu welcher - wohlgemerkt: ägyptischen - Kategorie ein Begriff gehört. Das kann bei der groben Bestimmung, was eine Droge ist, helfen. Das Pflanzendeterminativ kennzeichnet ein Wort als Pflanzenbezeichnung, ein Gefäßdeterminativ als Flüssigkeit, ein Körnerdeterminativ als Mineral oder etwas Körniges von Pflanzen oder Tieren. Und die Determinative sollte man ruhig ernst nehmen. Das hat Reymond leider oft nicht getan, wenn sie z.B. einen vagen Anklang eines demotischen Wortes an ein griechisches als Basis für deren Gleichsetzung nimmt. Passt dann aber das Determinativ des demotischen Wortes nicht, weil eine vermutete Pflanzenbezeichnung etwa mit dem Gefäßdeterminativ für Flüssigkeiten geschrieben wäre, schafft Reymond das Problem schnell dadurch aus der Welt, dass sie meint, dann sei eben im ägyptischen Text nicht die Pflanze selbst gemeint, sondern ein Extrakt aus ihr. ${ }^{26}$ So kann man sich natürlich alles passend machen. Lässt sich also sonst nichts zur Übersetzung eines Wortes sagen, so berücksichtige ich in der deutschen Wiedergabe wenigstens das Determinativ und schreibe z.B. „dysf-Körniges“, „" $m$-Pflanze“ usw. Damit orientiere ich mich an der Praxis des GRUNDRISSes, wo diese Determinierung eines ansonsten unklaren Wortes allerdings nur in Klammern in der Übersetzung zugesetzt wird nach dem Schema ,ps $\underline{d}$ (iii) “ oder ,šnf.t (iii)“. Ich halte dieses Verfahren für den Fachfremden weniger leicht eingängig als gleich ,ps $\underline{d}$-Körniges“ oder ,ک̌nf.t-Körniges“ zu schreiben. Bei Pflanzen macht es der Grundriss übrigens inkonsequenterweise genauso wie ich und schreibt z. B. ,innkPflanze“.27

Nun gibt es freilich eine zusätzliche Verkomplizierung. Es kann vorkommen, dass ein Ausdruck wie „schwarzer Stein“ ein Steindeterminativ nicht nur beim Wort für „Stein“ hat, sondern zusätzlich noch beim nachfolgenden Adjektiv „schwarz“. Dieses zweite Steindeterminativ bezieht sich also auf den ganzen zusammengesetzten Ausdruck

26 So z. B. REYMOND (1976): $149 \mathrm{zu}$ irs "Iris“ (tatsächlich ist $i \mathrm{RT}$ „MiLCH“ zu verstehen). Dabei wäre im Falle eines Pflanzensaftes nach den Regeln der demotischen Schrift das Normale, dass, wenn überhaupt, zum Pflanzendeterminativ noch ein Krugdeterminativ darugesetzt wird.

27 GRUNDRISS IV,1: 78. 
als einen Terminus „schwarzer Stein“ oder „Schwarzstein“. Allerdings ist es unmöglich, eine klare Grenze zwischen verdeutlichendem graphischen Zusatz durch den Schreiber und einem wirklichen zusammengesetzten, univerbierten Terminus zu ziehen. Denn in der demotischen Schrift ist es immer möglich, einem Adjektiv das Determinativ des Bezugswortes zu geben. Das ist ein vergleichsweise verbreitetes Phänomen, aber keine verpflichtende Regel. Meiner Einschätzung nach handelt es sich nicht einmal um ein überlieferungsgeschichtlich relevantes Detail, sondern um eine ad-hoc-Entscheidung der Schreiber. „Schwarzer Stein“ mit und ohne Gesamtdeterminativ dürfte stets dasselbe bezeichnen. Das eine als zusammengesetztes Wort, das andere als zwei getrennte zu werten, geht zu weit, schafft Probleme im Register und macht mindestens unnötig viele Verweise erforderlich. Um dennoch meinem Grundsatz, möglichst alle Informationen aus dem Original in die Übersetzung hinüberzuretten, treu zu bleiben, schreibe ich normal „schwarzer Stein“, wenn kein Gesamtdeterminativ folgt, aber „Schwarzer Stein“ mit großen „S“, wenn ein Gesamtdeterminativ dasteht. Im Kommentar zur Umschrift findet sich in diesem Fall der Hinweis auf die zusätzliche Determinierung.

Ein ganz hinterhältiges Problem beim Übersetzen fachsprachlicher Texte stellen in meinen Augen diejenigen Wörter dar, die in der Zielsprache als Fachtermini definiert sind. Als Ägyptologe stecke ich in dem Dilemma, übersetzen zu müssen, um den Text Nichtägyptologen verständlich zu machen. Aber ich kann nicht immer wissen, ob der in der Ägyptologie übliche Ausdruck vielleicht in der deutschen medizinischen, pharmazeutischen oder einer noch anderen Fachsprache als Fachterminus eine spezifische, definierte Bedeutung hat. Ich dürfte eigentlich z. B. im Zusammenhang mit einem antiken ägyptischen Text gar nicht von Rezepten sprechen. Denn modern wird unter einem Rezept eine ärztliche Anweisung zur Arzneianfertigung oder -ausgabe durch eine Apotheke verstanden. ${ }^{28}$ Apotheken oder überhaupt die Trennung von Arzt und Apotheker gab es aber in Ägypten nicht.

Ich finde, als Ägyptologe hat man auch ein Recht dazu, eine ägyptologische Übersetzung anzufertigen. Und man wird nicht umhinkönnen, vom Leser das Bewusstsein dafür zu verlangen, dass die Übersetzung eines ägyptischen medizinischen Textes - übrigens im

PSCHYREMBEL (2004): 1578. 
modernen Sinne auch nicht medizinisch - stärker erstens der ägyptischen, emischen und damit zweitens auch der ägyptologischen Begrifflichkeit verpflichtet sein muss als heutigen DIN-Normen und Definitionen. Solche etischen Begriffe anzuwenden muss einem Schritt nach dem Übersetzen vorbehalten sein, der übergreifenden Deutung und Beurteilung des Textes.

Wenden wir uns nun nicht nur der Wiedergabe der einzelnen Wörter, sondern des Textes in seiner Gesamtheit zu. Eine Aufgabe der Übersetzung muss es sein, die Struktur des Originaltextes zu verdeutlichen. Bezieht man sich auf die rein äußerliche Anlage, so ist, abgesehen vom Hinweis auf die Verwendung roter Tinte für die Überschriftenanfänge, nichts weiter zu sagen. Ansonsten wird im Wiener Text nämlich alles einfach fortlaufend hintereinander geschrieben; auch bei einem neuen Paragraphen wird keine neue Zeile begonnen. Dennoch halte ich es für wichtig, gerade angesichts der streng formalisierten, aber zu starker Abkürzung und oft zu einem Stichwortstil neigenden Sprache die inhaltliche Struktur zu verdeutlichen. Nehmen wir dieses Beispiel (3.23-3.24):

EIN ANDERES: schwarze Wicke; Menschen[mi]lch; MiLCH von Mohn. Zu ihm geben. EIN ANDERES: gekochter Wein; .[...]; (3.24) Rosen[ö]]. Erhitzen; $\mathrm{zu}$ ihm geben. EIN ANDERES: Beseitigen von Ohrstörung: [Sal]z; rtm-BaumSaft. Zu ihm GEBEN.

Da wir keine ägyptischen Muttersprachler sind und auch nicht wie ein ägyptischer Arzt tagtäglich in derartigen Texten lesen, erscheint es mir sinnvoll, die Teile Überschrift, Drogenliste und Handlungsanweisung deutlicher zu markieren, damit der moderne Leser sich besser in dem Text zurechtfinden kann. ${ }^{29}$ Ich benutze dazu Zeilenwechsel und für die Drogen Einrückungen: ${ }^{30}$

EIN ANDERES:

schwarze Wicke;

Menschen[mi]lch;

MiLCH von Mohn.

Zu ihm geben.

29 Aus Platzgründen ist für die auszugsweise Übersetzung in HOFFMANN und QUACK (2010): 300-305 lediglich jeder Rezeptanfang durch eine neue Zeile hervorgehoben.

30 Hier gegenüber der Edition in vereinfachter typographischer Gestaltung. 


\section{EIN ANDERES:}

gekochter Wein;

.[...];

(3.24) Rosen[öl].

Erhitzen; zu ihm geben.

EIN ANDERES: Beseitigen von Ohrstörung:

$[\mathrm{Sal}] \mathrm{z}$;

rtm-Baum-Saft.

Zu ihm GEBEN.

Gerade in stärker zerstörten Bereichen dürfte es sinnvoll sein, dem Leser eine derartige Orientierungshilfe an die Hand zu geben.

Eine weitere editorische Frage ist schließlich die nach der Platzierung des Kommentars. Zum Teil ist das natürlich bloß eine Geschmacksfrage. Aber man sollte es dem Leser nicht unnötig kompliziert machen. Ich selbst bevorzuge es, den Kommentar in Form von Fußnoten möglichst auf derselben Seite unterzubringen wie das Wort, auf das er sich bezieht. Aber es könnte auch sinnvoll sein, die Anmerkungen in einen eigenen Kommentarband auszulagern. Ich finde es jedenfalls unpraktisch, ständig blättern und womöglich auch noch überlegen zu müssen, ob man im philologischen Kommentar oder im Sachkommentar nachsehen muss. ${ }^{31}$ Ganz ungünstig für des Ägyptischen nicht Mächtige ist es meiner Meinung nach, wenn ein Kommentar nur über die originalsprachlichen Lemmata organisiert ist. Denn dann muss der Leser unter vielen anderen ihm nichts sagenden Stichwörtern das herausfinden, zu dem er gerade mehr erfahren möchte. Ich will aber keineswegs behaupten, den Stein der Weisen gefunden zu haben noch dass es ihn überhaupt gibt. Denn bei einem kurzen Text mag eine andere Organisationsform des Kommentars durchaus angemessener erscheinen als bei einem langen wie dem Wiener Papyrus.

31 Außerdem sind dabei verschiedene Varianten hinsichtlich der Reihenfolge denkbar: Erst die ganze Umschrift, dann der ganze philologische Kommentar, darauf die ganze Übersetzung, schließlich der ganze inhaltliche Kommentar? Oder doch lieber abschnittsweise Umschrift und Übersetzung zusammen, dann den Abschnittskommentar? Oder wie in der griechischen Papyrologie erst der ganze Text, knapp gehaltene Angaben zu Lesungen und Ergänzungen, dann die Übersetzung, endlich der ausführliche Kommentar? Es gibt natürlich noch mehr Möglichkeiten. 
Generell strebe ich an, meine Textedition möglichst mit dem GRUNDRISS kompatibel zu halten. Ich werde daher wie auch Reymond allgemeines Wortregister und Drogenregister wegen der speziellen Schwierigkeiten mit diesem Teil des Wortschatzes getrennt halten.

Ich verweise sowohl auf den Paragraphen als auch die Kolumne und Zeile, damit je nach Vorliebe des Lesers eine gesuchte Stelle sicher und schnell gefunden wird. Dabei stehen fett gedruckte Kolumnenund Zeilenzahl für einen an dieser Stelle komplett erhaltenen Beleg, normal gedruckte für einen beschädigten und eckige Klammern für einen ergänzten. Zu den im Original hieratischen Wörtern wird zusätzlich zum Faksimile auch die hieroglyphische Umschrift gegeben.

Beim Drogenregister werden die Belegstellen nach der Verwendung der Drogen aufgeschlüsselt. Um an dieser Stelle den raschen Zugriff auf möglichst viele Informationen zu ermöglichen, wird für jeden Beleg der Kontext des Schlüsselwortes auch in Umschrift und Übersetzung mitgeteilt. Jeder Eintrag wird dann noch in Anlehnung an das Drogenwörterbuch des GRUNDRISSes durch eine Diskussion zur Bedeutung abgeschlossen werden.

Es sollte selbstverständlich sein, dass in den Indizes auch wirklich das angegeben wird, was nach meiner Übersetzung und meinem Kommentar die Bedeutung eines Wortes ist oder sein könnte. Dabei sind auch eventuell bestehende Unsicherheiten kenntlich zu machen. Leider sieht man viel zu oft Register, in denen aus einer in der Diskussion im Textteil des Buches noch als fraglich erwogenen Bedeutung plötzlich eine sichere geworden ist, ohne Fragezeichen und ohne Hinweis auf die Übersetzungsproblematik oder Alternativen.

Ein Index, der die deutschen Drogennamen auflistet und auf die ägyptischen Bezeichnungen verweist, wird den Abschluss des Bandes bilden. Ich hoffe, dass ich damit den inhaltlichen Zugriff auf das Material erleichtere. Auch darin folge ich, anders als übrigens Reymond, dem Vorbild des GRUNDRISSes. 


\section{Literaturverzeichnis}

BAUM, Nathalie. 1999. „L'organisation du règne végétal dans l'Égypte ancienne et l'identification des noms de végétaux“. In: Sydney Aufrère (Hg.), Encyclopédie religieuse de l'Univers végétal. Croyances phytoreligieuses de l'Égypte ancienne, Bd. 1 (Orientalia Monspeliensia, 10). Montpellier: Université Paul Valéry: 421-443.

BAUMANN, Klaus-Dieter (Hg.). 2009. Translatologie aus integrativer Sicht: Übersetzungswissenschaftliche Analysen zwischen System und Globalität. Hamburg: Dr. Kovač.

Buber, Martin und Franz RosenzweIG. 1979-1985. Die Schrift. 4 Bde. Heidelberg: Schneider.

DePAUW, Mark. 1997. A Companion to Demotic Studies (Papyrologica Bruxellensia, 28). Brüssel: Fondation Égyptologique Reine Élisabeth.

Devauchelle, Didier und Michel Pezin. 1978. „Un papyrus médical demotique." Chronique d'Égypte 53: 57-66.

ERICHSEN, Wolja. 1954. Demotisches Glossar. Copenhagen: Munksgaard.

GERMER, Renate. 1985. Flora des pharaonischen Ägypten (Deutsches Archäologisches Institut Abteilung Kairo. Sonderschrift, 14). Mainz: von Zabern.

GerZyMisCH-ArBOGAST, Heidrun und Klaus MudersBaCH. 1998. Methoden des wissenschaftlichen Übersetžens (Uni-Taschenbücher, 1990). Tübingen/Basel: Francke.

GRUNDRISS = Grapow, Hermann, Hildegard von Deines und Wolfhart Westendorf. 1954-1973. Grundriss der Medizin der Alten Ägyter. 9 Bde. Berlin: Akademie-Verlag.

IV,1: Deines, Hildegard von, Hermann Grapow und Wolfhart Westendorf. 1958. Übersetzung der medizinischen Texte.

V: Grapow, Hermann. 1958. Die medizinischen Texte in bieroglyphischer Umschrift autographiert.

VI: Deines, Hildegard von, und Hermann Grapow. 1959. Wörterbuch der ägyptischen Drogennamen.

HoffmanN, Friedhelm. 1996. „Rezension zu Smith, M. 1993, The Liturgy of Opening the Mouth for Breathing." Bibliotheca Orientalis 53: 678-682.

HOFFMANN, Friedhelm. 2000. Aggpten. Kultur und Lebenswelt in griechisch-römischer Zeit. Eine Darstellung nach den demotischen Quellen (Studienbücher Geschichte und Kultur der Alten Welt). Berlin: Akademie-Verlag.

HOFFMANN, Friedhelm und Joachim Friedrich QUACK. 2010. „Demotische Texte zur Heilkunde“. In: Bernd Janowski und Gernot Wilhelm (Hgg.), Medizinische Texte (Texte aus der Umwelt des Alten Testaments. Neue Folge, 5). Gütersloh: Gütersloher Verlagshaus: 298-316.

LIPPERT, Sandra. 2004. Ein demotisches juristisches Lebrbuch. Untersuchungen zu Papyrus Berlin P 23575 rto. (Ägypologische Abhandlungen, 66). Wiesbaden: Harrassowitz. 
QUACK, Joachim Friedrich. 1999. (Rezension zu WESTENDORF, Wolfhart. 1999. Handbuch der altägyptischen Medizin) Orientalistische Literaturzeitung 94: 455-462.

PSCHYREMBEL. 2004. Klinisches Wörterbuch. 260. Aufl. Berlin/New York: de Gruyter.

REYMOND, Eva A. E. 1976. From the Contents of the Libraries of the Suchos Temples in the Fayyum. Teil 1: A Medical Book from Crocodilopolis. P. Vindob. D. 6257 (Mitteilungen aus der Papyrussammlung der Österreichischen Nationalbibliothek. Neue Serie, 10). Wien: Hollinek.

REYMOND, Eva A. E. 1983. „Demotic Literary Works of Graeco-Roman Date in the Rainer Collection of Papyri in Vienna“. In: Festschrift zum 100-jährigen Besteben der Papyrussammlung der Österreichischen Nationalbibliothek. Papyrus Erzherzog Rainer (P. Rainer Cent.). Wien: Hollinek: 42-60.

Vos, René L. 1993. The Apis Embalming Ritual. P. Vindob. 3873 (Orientalia Lovaniensia Analecta, 50). Leuven: Peeters.

$\mathrm{WB}=$ Erman, Adolf und Hermann Grapow. 1982. Wörterbuch der aegyptischen Sprache. 5 Bde. 4. Aufl. Berlin: Akademie-Verlag.

WESTENDORF, Wolfhart. 1999. Handbuch der altägyptischen Medizin. 2 Bde. (Handbuch der Orientalistik. Erste Abteilung, 36). Leiden/Boston/Köln: Brill. 